\title{
PREVISÃO DA VAZÃO AFLUENTE MENSAL DO RIO SÃO FRANCISCO USANDO-SE REDES DE ELMAN
}

\author{
Luiz Biondi Neto ${ }^{1}$ \\ Pedro Henrique Gouvêa Coelho ${ }^{1}$ \\ Luís Chiganer ${ }^{2}$ \\ Lídia Angulo Meza ${ }^{3}$ \\ Luiz Palmeira Monteiro ${ }^{4}$
}

\begin{abstract}
Resumo: Este artigo apresenta resultados de previsão da vazão afluente mensal do Rio São Francisco, que alimenta a usina de Sobradinho, usando-se redes neurais de Elman (RNA). As redes neurais de Elman utilizadas foram preparadas com o objetivo de receber como entrada amostras da série temporal da vazão afluente do Rio São Francisco deslocadas mês a mês. Para tal, nas camadas de entrada das referidas redes incluiu-se uma estrutura de atraso para que as redes recebessem os dados a serem processados em períodos de cinco anos, defasados mês a mês. A estrutura neural de Elman utilizada nesse trabalho é composta por até três camadas escondidas e uma camada de saída. Os dados usados para treinamento da rede foram os da série temporal das vazões afluentes medidas entre 1931 e 1991, devidamente pré-processadas. Os dados referentes aos anos de 1992 a 1996, e que não fizeram parte do treinamento da RNA, foram usados para testes de generalização da rede e na validação dos resultados, visto que os mesmos são conhecidos, fornecendo assim uma visão real do que aconteceu com a vazão afluente nos 60 meses entre 1992 a 1996. Os resultados obtidos indicam a adequação do uso das redes de Elman na previsão da vazão e o erro médio obtido foi menor que $0,2 \%$.
\end{abstract}

Palavras-chave: Previsão de Vazão, Redes Neurais de Elman, Redes Neurais Recorrentes.

Abstract: This paper shows results of monthly flow forecast for the São Francisco River using Elman neural networks. The used Elman Neural Networks had input layers suitably adjusted to process inputs consisting of monthly measured flow samples from São Francisco River. For that end, a delay structure was included in their input layer in order to the Elman neural network be able to process the data samples in 5 year periods, month by month. The used Elman neural networks architectures had up to three hidden layers and one output layer. The used data for training the neural networks comprised a 60 year period measured flows from 1931 to 1991, ready to be used in neural networks. Measured flow data from 1992 to 1996 were used for generalization tests and validation. The obtained results indicate that the used Elman neural networks are suitable for the monthly flow forecasting and the mean average error achieved was less than $0,2 \%$.

Keywords: Flow Estimation, Elman Neural Networks, Recurrent Neural Networks.

\footnotetext{
${ }^{1}$ Departamento de Engenharia Eletrônica e Telecomunicações - UERJ

${ }^{2}$ Departamento de Engenharia Elétrica - UERJ

${ }^{3}$ Departamento de Ciências dos Materiais, do Centro Tecnológico - UFF - Volta Redonda

${ }^{4}$ Departamento de Informática - UVA

ENGEVISTA, v. 7, n. 1, p. 63-72, abril 2005
} 


\section{INTRODUÇÃO}

A geração de energia elétrica no Brasil é predominantemente hidráulica, atualmente mesmo com a diversificação devido a criação de termelétricas a gás, nucleares, etc, mais de 85 \% da produção de energia elétrica ainda é hidrelétrica.

Assim o Brasil ocupa no mundo uma posição semelhante à da Arábia Saudita em petróleo. Nossa capacidade de geração baseia-se em dois elementos gratuitos, a água das chuvas e a força da gravidade.

Existem bacias hidrográficas compostas de centenas de rios permanentes e caudalosos e que se distribuem pelas regiões Norte, Nordeste, Sul, Sudeste e Centro-Oeste apresentando regimes de chuvas bem diferenciados.

Conseqüentemente, a geração de energia torna-se muito dependente da disponibilidade hídrica de médio e longo prazo dos reservatórios das usinas hidroelétricas instaladas no país no que tange a produção de energia firme ${ }^{5}$.

O sistema hidroelétrico brasileiro apresenta aspectos peculiares que o torna diferente dos outros sistemas de base hidroelétrica. Inicialmente, pelas características das afluências nos rios brasileiros, que apresentam acentuada sazonalidade 6 e elevado nível de incerteza, ao contrário do sistema do hemisfério norte no qual o regime hidrológico é proveniente, basicamente, do degelo. Além disso, pela característica de isolamento do sistema, que ao contrário dos demais sistemas hidroelétricos, não possuem interligação com sistemas vizinhos de base termoelétrica. E finalmente, porque apresenta forte acoplamento hidráulico entre suas unidades.

Assim, o planejamento (Eletropaulo, 1995), (Eletropaulo, 1996), (Eletropaulo,

\footnotetext{
${ }^{5}$ Energia firme é o maior valor possível de energia capaz de ser produzida continuamente pelo sistema, com as mesmas características do mercado, sem a ocorrência de déficits no caso de repetição das afluências do registro histórico.

${ }^{6}$ Sazonalidade é a tendência do processo em repetir um certo tipo de comportamento dentro de um período sazonal.
}

ENGEVISTA, v. 7, n. 1, p. 63-62, abril 2005
1997) da operação dessas usinas depende do conhecimento prévio do volume da água disponível nesses reservatórios, ou seja, é preciso conhecer a quantidade de água que estará disponível, com certa antecedência, para que se possa, finalmente, estimar a quantidade máxima de energia a ser gerada pela usina (Soares, 2001). Logo, em função dos bons resultados da previsão das vazões é possível realizar o planejamento energético de modo a atingir níveis ótimos de geração de energia.

Para viabilizar esse processo existem distribuídos em pontos específicos dos rios que integram as bacias hidrográficas, pontos de medição que fornecem medidas discretas da vazão, permitindo compor as séries históricas de vazão afluente. Prever a vazão significa descobrir, por antecipação, os valores da quantidade de água que irá afluir nesses pontos de medição com base nas séries históricas disponíveis (Chatfield ,1991).

A previsão de vazão é um verdadeiro desafio utilizado para o gerenciamento dos recursos hídricos numa bacia (Moraes, 1995), (Moraes, 1996). A previsão de inundações, da umidade do solo para agricultura, os níveis de navegação de uma via, a disponibilidade hídrica para abastecimento de água, irrigação e produção de energia são usos conhecidos para a previsão antecipada da vazão numa bacia hidrográfica (Tucci, 2002).

A previsão de vazão pode ser de curto, médio e longo prazo (Tucci, 2002). A previsão de curto prazo é usada para estimar a vazão num trecho da bacia com antecedência de horas ou poucos dias (Tucci, 2002). A previsão de médio prazo envolve a previsão de vazão com antecedência de um a alguns meses e depende, fortemente, das condições conhecidas de clima e dos oceanos que podem influenciar as vazões futuras na bacia hidrográfica (Tucci, 2002).

A previsão de longo prazo é a estimativa, normalmente estatística, dos riscos de ocorrência de vazões num determinado local, como por exemplo: o 
risco de inundação numa seção de um rio, a probabilidade de períodos úmidos e secos, dentre outros (Tucci, 2002).

Tradicionalmente o setor elétrico usa para prever a vazão afluente, o método de Box-Jenkins (Box, 1976), (Hoff, 1983), que pressupõe uma relação linear entre os valores presente e passado da vazão. Podem ser aliados aos modelos lineares autoregressivos (AR), de média móvel (MA) e o autoregressivo de média móvel (ARMA), que igualmente não são os mais adequados para tratar um conjunto de dados com características não lineares e não estacionárias (Chatfield, 1991) inerentes às séries de vazão.

Por outro lado, as redes neurais artificiais (RNAs) (Fog, 1995), (Lachtermacher, 1995), (Sarle, 1995) são modelos compostos de um certo número de elementos não lineares (neurônio artificial) operando em paralelo e dispostos em camadas similarmente às redes neurais biológicas e através de algoritmos específicos as RNAs podem aprender certo conhecimento (Haykin, 1994), (Evans, 1991), (Siqueira, 2002). As RNAs podem ser grupadas em duas categorias: redes sem realimentação (feedforward networks) e com realimentação (recurrent networks). As redes sem realimentação são estáticas, isto é uma dada entrada pode produzir apenas um conjunto de saída e, portanto não produzem nenhum tipo de memória.

Por outro lado as redes recorrentes permitem que as características temporais da informação sejam memorizadas. Um caso típico é a rede de Elman (Elman, 1990), parcialmente recorrente, e que será usada para fazer a previsão da vazão nessa pesquisa.

As principais características no uso dessa metodologia na análise de séries temporais são os seguintes:

- As RNAs são auto-monitoráveis, isto é, apreendem como realizar previsões precisas.

- As RNAs permitem previsões iterativas.
- As RNAs são capazes de lidar com a não estacionariedade e a não linearidade das séries temporais investigadas.

- As RNAs oferecem predição paramétrica e não paramétrica.

Diversos profissionais têm se destacado nessa área: $\mathrm{O}$ pesquisador Zurada (Zurada, 1997) introduz o conceito de construção de redes seqüenciais, usando uma rede parcialmente recorrente de Elman.

O pesquisador Aquino (Aquino, 1999) aplica Redes Neurais Artificiais no Planejamento da Operação de Sistemas Hidrotérmicos de Geração.

O pesquisador Milioni (Milioni, 2000) apresenta pesquisa cujo objetivo é o de tentar abstrair a natureza física do processo, simplificando a abordagem, através de um sistema que utilize, numa primeira etapa, modelos econométricos de regressão múltipla que expliquem a vazão de uma seção de controle a partir da observação do nível do rio a montante.

O pesquisador Tucci (Tucci, 2001) apresenta os resultados da previsão em tempo real do volume afluente ao reservatório de Ernestina.

Este trabalho tem como objetivo investigar a previsão da vazão afluente, num período de 5 anos mês a mês de 1992 a 1996 (60 meses), visando auxiliar a decisão dos especialistas do setor elétrico, envolvidos em planejamento energético.

Não é objetivo do presente trabalho, investigar os processos de préprocessamento (Chatfield, 1991), (Box, 1976), (Hoff, 1983), (Moraes, 1996), usados, normalmente, no tratamento de séries temporais e consequentemente nesse trabalho.

A principal motivação está ligada ao fato da existência de um excedente de energia não devidamente aproveitada, isto é uma diferença entre a geração média em todo o histórico de vazões (média e longo prazo) e a energia firme, não devidamente aproveitada. 
O trabalho será dividido em 5 seções. A primeira faz uma introdução sobre 0 assunto ressaltando o estado da arte.

$\mathrm{Na}$ seção 2 são mostrados os fundamentos teóricos de redes neurais parcialmente recorrentes de Elman. Em 3 é feita a descrição e a modelagem do problema.

Na seção 4 são mostrados os resultados relativos a implementação da rede e finalmente em 5 encontram-se as conclusões.

\section{ELEMENTOS BÁSICOS}

As redes neurais estáticas como é o caso dos Perceptrons de múltiplas camadas (MLP), treinadas normalmente pelo algoritmo de retropropagação de erro (Cichocki,1996), apresentam certa dificuldade em realizar mapeamentos dinâmicos (Haykin, 1994).

Conseqüentemente aprender as características temporais de um sinal, que represente a série histórica da vazão afluente de um rio, pode se tornar uma tarefa difícil.

Para resolver o problema pode-se usar uma rede MLP tradicional, porém com as entradas atrasadas no tempo. A Figura 1 mostra esse esquema denominado de rede temporal, no qual uma rede MLP é alimentada por uma entrada $u(t)$ que é sucessivamente atrasada de uma unidade pela estrutura $\mathrm{z}^{-1}$ até, genericamente, $\mathrm{u}(\mathrm{t}-\mathrm{k})$ e que tem como saída a $\mathrm{y}(\mathrm{t})=\mathrm{u}(\mathrm{t})$.

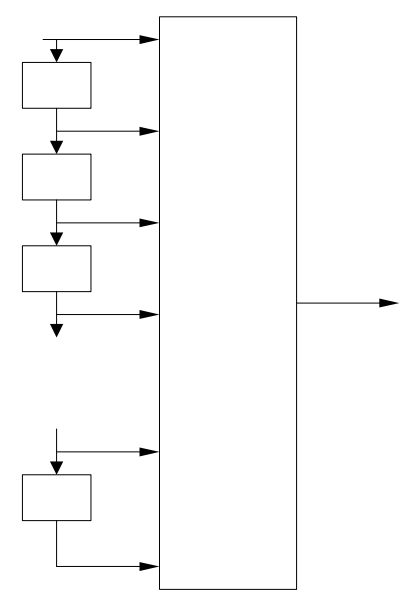

Figura 1 - Rede Temporal
Nesse caso, o atraso está sendo aplicado apenas na entrada, porém poderia ser aplicado também nas camadas escondidas e na saída.

Uma outra forma de resolver o problema é usando-se redes com elos de realimentação, denominadas de redes recorrentes.

Normalmente as redes recorrentes podem incorporar uma MLP ou parte dela, fazendo uso de sua capacidade de mapeamento não linear.

De forma geral as redes recorrentes, são muito adequadas para tratar problemas temporais, pois as conexões recorrentes ajudam a rede a memorizar as informações atuais no momento seguinte da seqüência investigada.

Redes neurais recorrentes podem apresentar uma ou mais malhas de realimentação. Naquelas totalmente recorrentes tem-se cada neurônio conectado a qualquer dos outros e constituem o caso mais geral de redes neurais artificiais.

Uma maneira elegante de se representar uma rede neural recorrente é através de um modelo em espaço de estados. A noção de estado representa um papel vital na formulação matemática de um sistema dinâmico. O estado de um sistema dinâmico é formalmente definido como um conjunto de quantidades que sintetiza toda a informação sobre o comportamento passado do sistema que é necessário para descrever unicamente seu comportamento futuro, exceto pelos efeitos puramente externos advindos da entrada ou excitação aplicada. Seja o vetor de dimensão [q x 1] $\mathbf{x}(\mathrm{k})$ o estado de um sistema não linear discreto. Seja o vetor de dimensão [m x 1] $\mathbf{u}(\mathrm{k})$ a entrada aplicada ao referido sistema, e o vetor $\mathbf{y}(\mathrm{k})$ a sua saída correspondente. Em termos matemáticos, o comportamento dinâmico do sistema, sem ruído, pode ser descrito pelo seguinte par de equações não lineares.

$$
\begin{aligned}
& \mathbf{x}(k+1)=\varphi\left(W_{a} \mathbf{x}(k)+W_{b} \mathbf{u}(k)\right) \\
& \mathbf{y}(k)=C \mathbf{x}(x)
\end{aligned}
$$


onde $W_{\mathrm{a}}$ é uma matriz de dimensão [q x q], $W_{\mathrm{b}}$ é uma matriz de dimensão [q x m], $C$ é uma matriz de dimensão [p $\mathrm{x}$ q], e $\varphi: \Re^{q} \rightarrow \Re^{q}$ é um mapeamento descrito por:

$\varphi:\left[\begin{array}{l}x_{1} \\ x_{2} \\ \cdot \\ \cdot \\ \cdot \\ x_{q}\end{array}\right] \rightarrow\left[\begin{array}{l}\varphi\left(x_{1}\right) \\ \varphi\left(x_{2}\right) \\ \cdot \\ \cdot \\ \varphi\left(x_{q}\right)\end{array}\right]$

para alguma não linearidade sem memória $\varphi: \quad \mathfrak{R} \rightarrow \mathfrak{R}$ atuando componente a componente.

Os espaços $\mathfrak{R}^{\mathrm{m}}$, $\mathfrak{R}^{\mathrm{q}}$, e $\mathfrak{R}^{\mathrm{p}}$ são chamados de espaços de entrada, de estado e de saída respectivamente. A dimensão do espaço de estado, q, constitui a ordem do sistema.

Dessa forma a rede neural recorrente representada pelas equações (1) e (2) é um sistema dinâmico com $\mathbf{m}$ entradas e $\mathbf{p}$ saídas de ordem q. A equação (1) forma a equação de processo do modelo e (2) é a equação de medida. Com relação às matrizes $\mathbf{W}_{\mathbf{a}}, \mathbf{W}_{\mathbf{b}}$ e $\mathbf{C}$, e a função não linear $\varphi($.$) pode-se tecer as considerações a$ seguir.

A matriz $\mathbf{W}_{\mathbf{a}}$ contém os pesos sinápticos dos q neurônios que são conectados aos nós de realimentação na entrada. A matriz W bontém os pesos sinápticos desses neurônios que são conectados aos nós fontes também na entrada. A matriz $\mathbf{C}$ indica a forma da combinação linear dos estados que caracterizarão a saída da rede neural. A função não linear $\varphi($.) caracteriza a função de ativação de qualquer neurônio na rede. Esta função de ativação é muitas vezes definida pela função tangente hiperbólica (4).

$$
\varphi(x)=\tanh (x)=\frac{1-e^{-2 x}}{1+e^{-2 x}}
$$

Uma propriedade importante de uma rede neural recorrente descrita pelas ENGEVISTA, v. 7, n. 1, p. 63-72, abril 2005 equações de espaço de estado (1) e (2) é a sua capacidade de aproximar uma classe ampla de sistemas não lineares dinâmicos.

A figura 2 representa uma rede neural recorrente com três entradas, três estados e uma saída, ou seja, $\mathrm{m}=3, \mathrm{q}=3$ e $\mathrm{p}=1$.

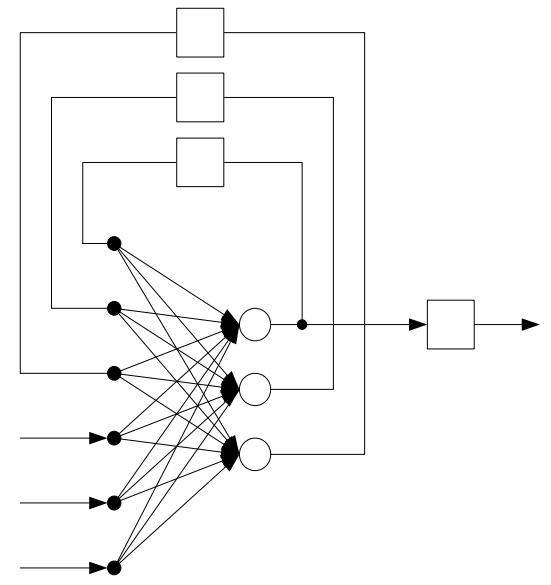

Figura 2 - Rede Totalmente Recorrente

As matrizes $\mathbf{W}_{\mathbf{a}}$ e $\mathbf{W}_{\mathbf{b}}$ são definidas como:

$$
\begin{aligned}
& W_{a}=\left[\begin{array}{lll}
w_{11} & w_{12} & w_{13} \\
w_{21} & w_{22} & w_{23} \\
w_{31} & w_{32} & w_{33}
\end{array}\right] \\
& W_{b}=\left[\begin{array}{lll}
w_{14} & w_{15} & w_{16} \\
w_{24} & w_{25} & w_{26} \\
w_{34} & w_{35} & w_{36}
\end{array}\right]
\end{aligned}
$$

A matriz $\mathbf{C}$ é um vetor linha definido por:

$$
C=\left[\begin{array}{lll}
1 & 0 & 0
\end{array}\right]
$$

A Figura 3 representa um modelo autoregressivo não linear com entradas exógenas (Haykin, 1994). Apresenta uma memória de linha de atraso com $n$ unidades que é aplicada à entrada. A saída também é realimentada à entrada por outra memória de atraso. 


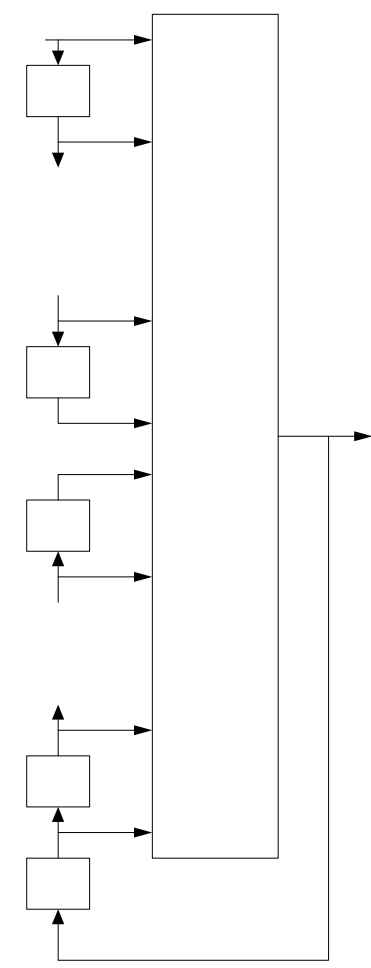

Figura 3 - Modelo auto-regressivo

Para uma entrada $\mathrm{u}(\mathrm{t})$ a saída $\mathrm{y}(\mathrm{t}+1)$ estará adiantada de uma unidade em relação à entrada.

Os valores atuais e passados da entrada são: $u(t), u(t-1) \quad u(t-2) \quad \ldots \quad u(t-k+1)$ e os valores atrasados da saída são: $\mathrm{y}(\mathrm{t}), \mathrm{y}(\mathrm{t}-1)$ $\mathrm{y}(\mathrm{t}-2) \ldots \mathrm{y}(\mathrm{t}-\mathrm{k}+1)$ sobre os quais é feita a regressão da saída do modelo, onde $\mathfrak{I}$ é uma função não linear.

$$
\begin{aligned}
& \mathrm{y}(\mathrm{t}+1)= \mathfrak{T}(\mathrm{y}(\mathrm{t}), \ldots, \mathrm{y}(\mathrm{t}-\mathrm{k}+1), \\
&\mathrm{u}(\mathrm{t}), \ldots \mathrm{u}(\mathrm{t}-\mathrm{k}+1))
\end{aligned}
$$

A rede de Elman, que será usada nessa pesquisa, é uma arquitetura de RNA considerada parcialmente recorrente, pois os elos de realimentação ocorrem entre a saída e a entrada da primeira camada oculta.

O elo recorrente é feito através de uma unidade de contexto, normalmente uma estrutura de atraso do tipo $\mathrm{z}^{-1}$, armazenando a saída da primeira camada oculta por um passo de tempo. Assim, essa estrutura garante a geração de padrões variáveis no tempo, habilitando essa configuração neural para aplicações envolvendo séries temporais.
Além da camada recorrente a rede pode apresentar várias outras camadas formando uma tradicional MLP, com uma ou várias saídas.

O algoritmo usado para o treinamento é o de retropropagação de erro (backpropagation), extensivamente discutido na literatura (Haykin, 1994) e (Cichocki, 1996). Na verdade existe apenas um du(da)lo que deve ser tomado na aplicação do algoritmo. Num dado instante a rede recebe não só as entradas externas, normalmente aplicaß̧ăs ta ela, mas também as realimentadas da saída da primeira camada oculta, obtidas no instante anterior.

A figura $4 \mathrm{t}$ apresenta uma rede de Elman, na qual se procura mostrar, com nitidez, o mecanismo de funcionamento da estrutura de realimentação.

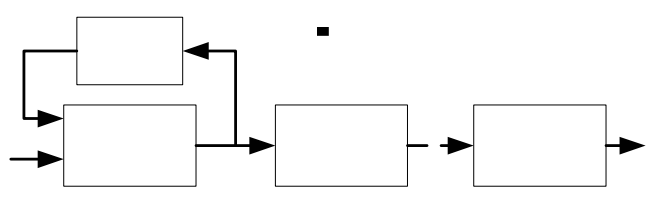

\section{telftrrak 4 R Rde Neural de Elman}

No caso da utilizaç̃̃o em previsão de séries temporais é possível adotar soluções híbridas, fazendo-se com as entradas sejam devidamente atrasadas, conforme a figura

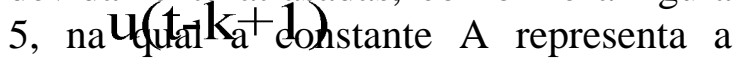
defasagem máxima.

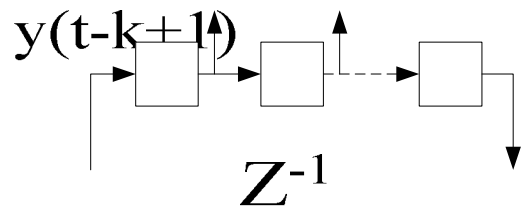

Figura 5 - Malha defasadora da entrada

\section{3 МФQНА-АОЕМ}

Neste trabalho adotaremos um modelo semelhante ao mostrado na figura 4 , no qual as entradas são apresentadas a rede de 60 em 60 meses e encontram-se defasadas uma da outra de 1 mês, conforme malha defasadora representada na figura 5.

$$
y(t-1)
$$

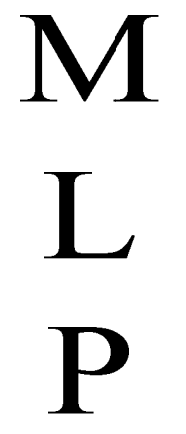


Nossa disponibilidade de dados é de 65 anos de vazão mensal relativa a vazão afluente do rio São Francisco. Desse total usaremos para treinamento da rede os dados referentes a 60 anos (1931 a 1991) de vazão, deixando para teste de generalização e validação os 5 anos restantes (1992 a 1996). Os dados de treinamento estão arranjados segundo uma matriz de entrada contendo 661 linhas cada uma defasada da outra de 1 mês e 60 colunas relativas ao período de 5 anos. $\mathrm{O}$ vetor alvo terá 60 elementos defasados de um mês em relação a última linha da matriz de entrada.

A figura 6 mostra apenas o modelo da rede utilizada, sem a malha defasadora, representada na figura 5. A matriz de teste de generalização e validação dos resultados é construída de forma semelhante a matriz de entrada.

O número ótimo de neurônios nas diversas camadas escondidas (N...R), vistos na mesma figura, é escolhido durante o treinamento, visando-se obter a melhor performance da rede, especialmente no tocante a generalização ${ }^{7}$.

A camada de saída tem um único neurônio que apresentará o vetor com 60 elementos representativos da vazão que se deseja prever, isto é de 5 anos mês a mês.

$\mathrm{O}$ algoritmo de treinamento utilizado foi o de retropropagação de erro adequado á essa arquitetura de rede.

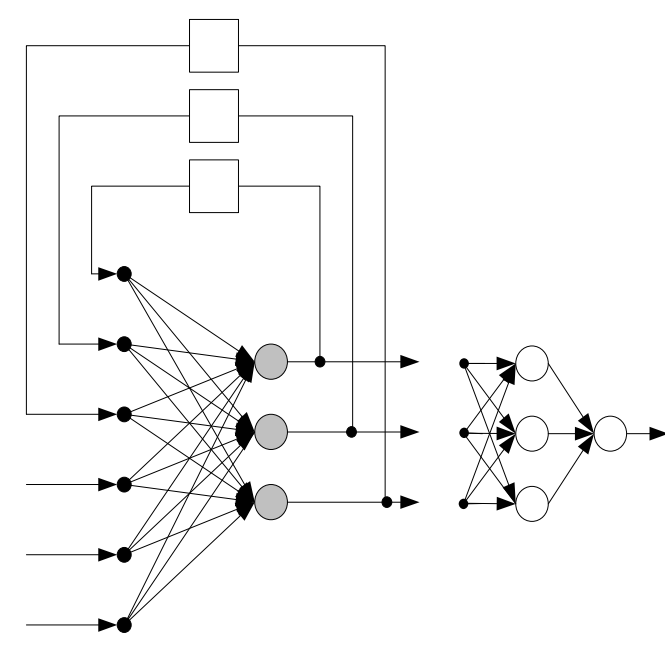

Figura 6 - Modelo da rede investigada

\section{RESULTADOS}

Visando-se obter as melhores características de generalização foram testadas diversas arquiteturas, usando-se os dados conhecidos referentes aos anos de 1992 a 1996 (60 meses) e que por questões obvias, não tomaram parte no treinamento da rede. A arquitetura que resultou na melhor performance engloba três camadas ocultas (357-186-51) e uma camada de saída, com apenas um neurônio, e usando a função tangente hiperbólica como função de ativação. $O$ método numérico usado para a minimização do erro foi o do gradiente decrescente, com taxa de aprendizado adaptativa e coeficiente de momento, que minimiza as oscilações. A convergência ocorreu decorrida 1118 épocas para uma tolerância de erro de $10^{-5}$ conforme a curva de treinamento representada na figura 7 .

Depois de devidamente treinada a arquitetura, os parâmetros de treinamento e as matrizes de pesos foram armazenados em arquivo próprio para futuramente serem utilizados na previsão mês a mês e no prazo de 5 anos.

\footnotetext{
${ }^{7}$ Generalização é a capacidade intrínseca da RNA em responder adequadamente a padrões que não fizeram parte do treinamento. 


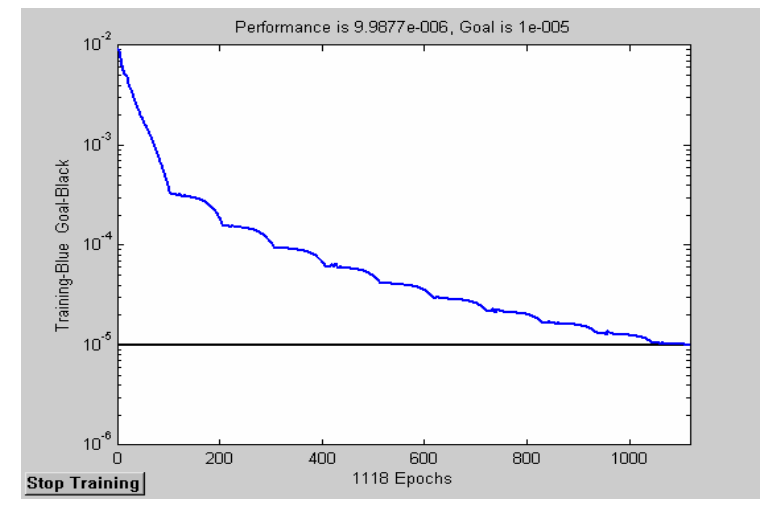

Figura 7 - Curva de treinamento

A figura 8 apresenta a curva da vazão conhecida (contínua), referente aos anos entre 1992 a 1996, (60 meses). Superposta a esta curva encontra-se também a curva de previsão (pontilhada) obtida mês a mês pela RNA, apresentando um erro médio menor que $0.2 \%$.

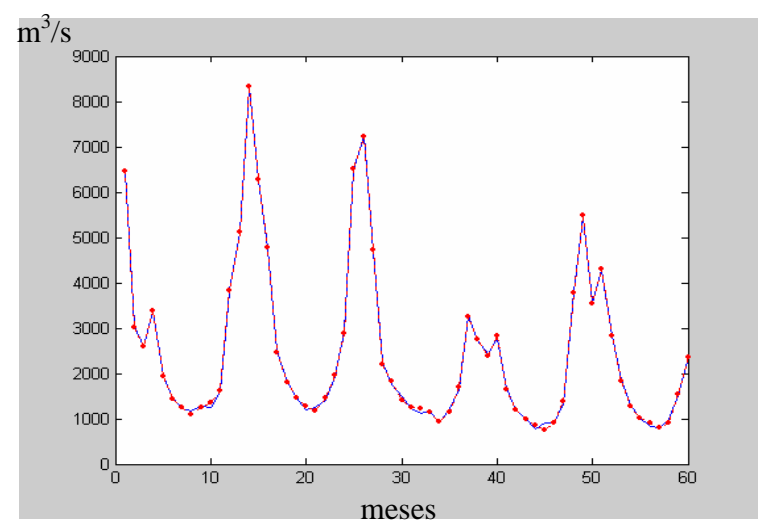

Figura 8 - Previsão

A figura 9 representa a curva de erro percentual de previsão feita pela RNA, cujo valor médio não ultrapassa $0.2 \%$.

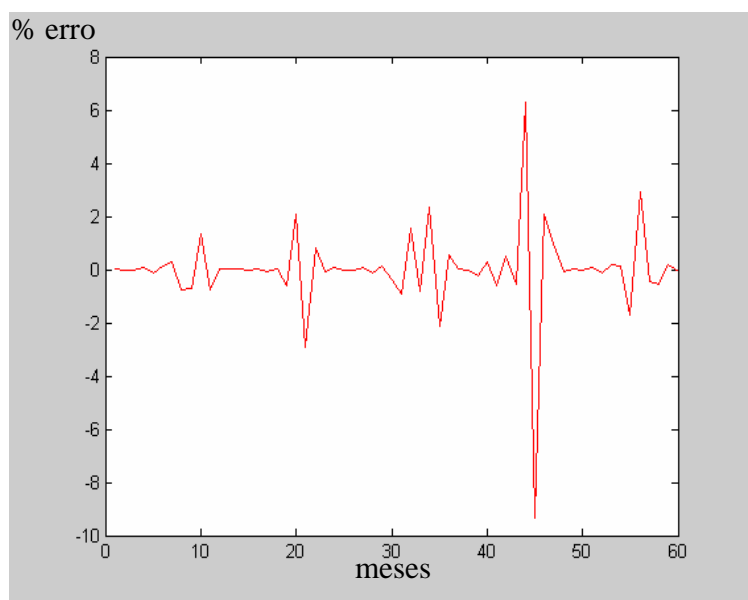

Figura 9 - Erro percentual

\section{CONCLUSÕES}

O resultado obtido através da metodologia proposta no presente artigo revela que o uso de redes parcialmente recorrentes de Elman, com malha defasadora na entrada, parecem ser adequadas para previsão de séries temporais.

No caso investigado, o erro médio de previsão de cerca de $0.2 \%$ é muito menor que os obtidos por RNAs tradicionais usando-se "janelamento", que atingem a casa de 5\% (Lachtermacher, 1995), (Chakraborty, 1992). Quando comparado com o erro obtido por processos puramente estatísticos, como é o caso do Box-Jenkins (Box, 1976), (Hoff, 1983) e suas variações onde o erro médio é maior que $10 \%$, a superioridade da rede de Elman ainda é mais significativa.

Finalmente, se forem incorporadas ao processo às características inerentes a temperatura do oceano como mais uma entrada da RNA estamos certos que os resultados ainda serão melhores. Infelizmente, no momento só dispomos dessas temperaturas para uma parte do período investigado.

Mesmo assim, simulações preliminares mostram resultados que justificam nosso empenho em obter a série completa da temperatura do oceano.

Dessa forma fica nítida a indicação de que vale a pena investigar mais sobre comportamento das redes de Elman e suas variações na previsão de séries temporais.

\section{AGRADECIMENTOS}

Este trabalho teve o apoio do Fundo Setorial de Energia (CT-Energ), por intermédio do CNPq (CT-Energ/CNPq 01/2003), processo 400646/2003-0

\section{REFERÊNCIAS}

Cichocki, A., Unbehauen, R., 1996. Neural Networks for Optimisation and Signal Processing, New York, USA, John Wiley \& Sons, Inc.

Tucci, C. E. M., Robin T. C., Dias P. L. da S., Collischonn W., 2002. Previsão de 
médio prazo da afluência de reservatórios com base na previsão climática. In Relatório Final Projeto: BRA/00/029, Instituto de Astronomia, Geofísica e Ciências Atmosféricas Universidade de São Paulo e Instituto de Pesquisas Hidráulicas Universidade Federal do Rio Grande do Sul.

Box, G. E. P., and Jenkins, G. M., 1976. Time Series Analysis: Forecasting and Control. California, USA. San Francisco: Holden Day, 2nd. ed.

Fog, T.L. et al, 1995. Training and Evaluation of Neural Networks for Multi-Variate Time Series Processing. In Proceedings of IEEE International Conference on Neural Networks. IEEE Press.

Lachtermacher, G. and Fuller, J.D., 1995. Backpropagation in Time series Forecasting. In Journal of Forecasting. Vol 14, 381-393.

Sarle, W.S., 1995. Stopped Training and other remedies for Overfitting. In Proceedings of the 27th Symposium on the Interface.

Eletropaulo, 1995. Rios / Reservatórios / Enchentes. In Departamento de Patrimônio Histórico. Historia $e$ Energia, v. 5.

Eletropaulo, 1996. O Capital Privado na Reestruturação do Setor Elétrico Brasileiro. In Departamento de Patrimônio Histórico. Historia $e$ Energia, v. 6.

Eletropaulo, 1997. Estatização versus Privatização. In Departamento de Patrimônio Histórico. Historia $e$ Energia, v. 7.

Moraes, J. M., Genovez, A.M., Mortatti, J., Pellegrino, G., Ballester, M.V., Martinelli, L. A, 1996. Análises e Modelagem de uma Série Temporal de Vazão sob a Influência de Ações Naturais e Antrópicas. In Anais do XVII Congresso Latino Americano de Hidraúlica.
Moraes, J. M., Pellegrino, G., Ballester, M. V., Martinelli, L. A., Victoria, R. L., 1995. Hydrological Parameters of a Southern Brazilian Watershed and its Relation to Human Induced Changes. In Annales of 20th General Assembly of the European Geophyscial Society, v13: 506-507.

Chatfield E., 1991. The Analysis of Time Series, New York, USA, Chapman and Hall, fourth edition.

Chakraborty, K., Mehrota, K., Chilukuri, K. M., and Ranka, S., 1992. Forecasting the Behaviour of Multivariate Time Series Using Neural Networds. In Neural Networks, v5, 961-970.

Evans, R. M. and S. Alvin, J., 1991. Relating Numbers of Processing Elements in a Sparse Distributed Memory Model to Learning Rate and Generalization, In ACM APL Quote Quad, v21(4), 166-173.

Soares F., S., Bessa, M. R., 2001. Otimização da Operação de Reservatórios de superfície: Um estudo comparativo entre diferentes metodologias aplicadas ao sistema de Furnas, In XVI SNPTEE.

Siqueira, T. G., Soares F., S., 2002. Aplicação de Redes Neurais com função de ativação de Base Radial na predição de séries temporais nãoestacionárias, In XIV Congresso Brasileiro de Automação.

Hoff C. J., 1983. A Practical Guide to Box-Jenkins Forecasting, Belmont, CA., USA, Lifetime Learning Publications.

Elman J. L., Finding Structure in Time, 1990. In Cognitive Science, vol. 14, pp. 179-211.

Tucci, C. E. M., Brun, G., 2001. Previsão em tempo real do volume afluente ao reservatório de Ernestina. In Revista Brasileira de Recursos Hídricos. V.6, n.2, p.73 - 79 .

Aquino, R., B. de, Carvalho, M., A., Jr., Souza, B., A., 1999. Redes Neurais Artificiais: Uma Aplicação ao Planejamento da Operação de Sistemas Hidrotérmicos de Geração. In Proceedings of the IV Brazilian 
Conference on Neural Networks - IV Congresso Brasileiro de Redes Neurais, pp. 164-169.

Zurada J. M. and Cholewo T., J., 1997. Sequential Network Construction for Time Series Prediction. In Proceedings of the IEEE International Joint Conference on Neural Networks, pp 2034-2039.

Milioni, A., Z., e Acioli A., O., 2001. Modelos Econométricos para a Previsão de Cheias Fluviais . In Simpósio Brasileiro de Pesquisa Operacional.

Haykin S. , 1994. Neural Networks, a comprehensive Foundation; London, Macmillan College Publishing Co. 